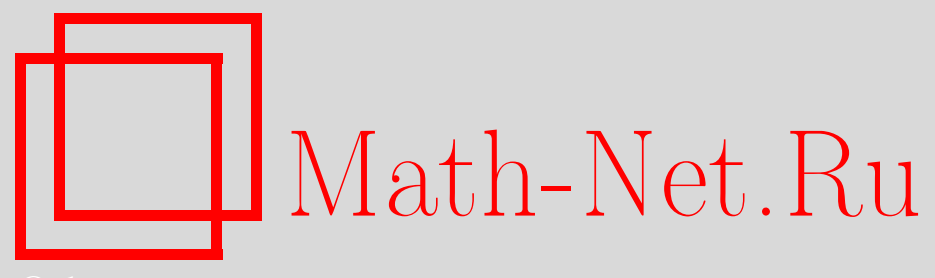

О. А. Козлитин, Использование 2-линейного регистра сдвига для выработки псевдослучайных последовательностей, $M a-$ тем. вопр. криптогр., 2014, том 5, выпуск 1, 39-72

DOI: https://doi.org/10.4213/mvk106

Использование Общероссийского математического портала Math-Net.Ru подразумевает, что вы прочитали и согласны с пользовательским соглашением http: //www . mathnet.ru/rus/agreement

Параметры загрузки:

IP : 54.162 .85 .209

26 апреля 2023 г., 12:31:07

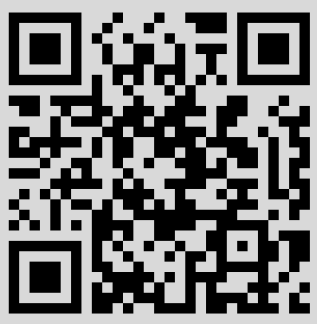




\title{
Использование 2-линейного регистра сдвига для выработки псевдослучайных последовательностей
}

\author{
О. А. Козлитин \\ ООО «Центр сертификационных исследований», Москва
}

Получено 22.IV.2013

В работе описаны периодические свойства почти всех 2-линейных рекуррент, вырабатываемых 2-линейным регистром сдвига с равными характеристическими многочленами максимального периода. Предложен класс нелинейных функций самоуправления, обеспечивающих наличие в графе переходов состояний циклов максимально возможной длины. Описаны линейные функции выхода, сохраняющие период последовательности состояний.

Ключевые слова: линейная рекуррентная последовательность, 2-линейный регистр сдвига, функция самоуправления

Constructing pseudorandom sequences by means of 2-linear shift register

\section{O. A. Kozlitin}

\section{LLC "Sertification Research Center", Moscow}

\begin{abstract}
We describe the periodicity properties for almost all 2-linear recurrent sequences generated by 2-linear shift register with identical connection polynomials of maximal period. A class of self-control nonlinear functions are suggested such that the existence of maximally possible cycles in a transition graph of states is guaranteed. Linear output functions preserving the period of sequence are described.
\end{abstract}

Key words: linear recurrent sequence, 2-linear shift register, self-control function

Citation: Mathematical Aspects of Cryptography, 2014, vol. 5, no. 1, pp. 39-72 (Russian). 


\section{1. Введение}

Информатизация общества, развитие техники связи и связанный с ними рост трафика в компьютерных сетях ставят новые задачи в сфере информационной безопасности. Важное место в арсенале средств защиты данных занимает криптография, причем в последнее время увеличивается значение такого параметра, как быстродействие (пропускная способность) шифров. Растет интерес к поточным криптосистемам, т. е. к системам, реализующим при каждом фиксированном значении ключа последовательность обратимых отображений (вообще говоря, различных), действующую на последовательность блоков открытого текста [13].

Основой всякой поточной криптосистемы является генератор псевдослучайных последовательностей (ПСП), качеством которого во многом определяются свойства системы в целом. Разработка генераторов ПСП, построенных на новых математических принципах, - важная прикладная задача, которой уделяется значительное внимание за рубежом. Свидетельство тому - конкурс eStream, проводившийся в 2004-2008 гг. в целях создания новых европейских стандартов поточного шифрования. Одним из победителей конкурса в номинации «Поточные шифры для программного применения с большой пропускной способностью» стала предложенная в 2005 году криптосистема Salsa20. Автор схемы Daniel J. Bernstein построил генератор ПСП на базе так называемого двумерного регистра сдвига с нелинейными функциями обратной связи [19].

Двумерный регистр сдвига является естественным обобщением стандартного (одномерного) регистра сдвига [13]. Его простейшая разновидность - двумерный линейный регистр сдвига (2-ЛРС; в англоязычной литературе используется аббревиатура 2-LFSR: 2-linear feedback shift register). Неформально этот автомат можно описать следующим образом.

Пусть $R$ - конечное коммутативное кольцо с единицей $1, F(x)$ и $G(x)-$ унитарные (со старшим коэффициентом 1) многочлены над $R$ степеней $m_{0} \geq 1$ и $m_{1} \geq 1$ соответственно, $R_{m_{0}, m_{1}}-$ множество всех прямоугольных матриц размерами $m_{0} \times m_{1}$ над кольцом $R, w(0) \in R_{m_{0}, m_{1}}, \mathfrak{F}=\left\{0,1, \ldots, m_{0}-1\right\} \times$ $\times\left\{0,1, \ldots, m_{1}-1\right\}$. Рассмотрим отображение $u: \mathbb{N}_{0}^{2} \rightarrow R$ такое, что в таблице

\begin{tabular}{|c|c|c|c|c|c}
\hline$u(0,0)$ & $u(0,1)$ & $u(0,2)$ & $\ldots$ & $u(0, j)$ & $\ldots$ \\
\hline$u(1,0)$ & $u(1,1)$ & $u(1,2)$ & $\ldots$ & $u(1, j)$ & $\ldots$ \\
\hline$u(2,0)$ & $u(2,1)$ & $u(2,2)$ & $\ldots$ & $u(2, j)$ & $\ldots$ \\
\hline$\ldots$ & $\ldots$ & $\ldots$ & $\ldots$ & $\ldots$ & $\ldots$ \\
\hline$u(i, 0)$ & $u(i, 1)$ & $u(i, 2)$ & $\ldots$ & $u(i, j)$ & $\ldots$ \\
\hline$\ldots$ & $\ldots$ & $\ldots$ & $\ldots$ & $\ldots$ & $\ldots$ \\
\hline
\end{tabular}


каждый столбец есть линейная рекуррентная последовательность (ЛРП) с характеристическим многочленом $F(x)$, а каждая строка - ЛРП с характеристическим многочленом $G(x)$, причем на пересечении первых $m_{0}$ строк и первых $m_{1}$ столбцов стоит матрица

По таблице в соответствии с некоторым законом движется прямоугольное окно размерами $m_{0} \times m_{1}$. В начальный момент времени окно располагается в левом верхнем углу таблицы. Текущее заполнение окна w $(t)$ считается текущим заполнением регистра. Следующий рисунок иллюстрирует понятие 2-линейного регистра сдвига (здесь $R^{\left(m_{0}\right)}-$ модуль вектор-столбцов длины $m_{0}$ над $R$, а $R^{m_{1}}$ - модуль вектор-строк длины $m_{1}$ над $R$ ):

Первоначально 2-ЛРС возник в теории кодирования. В 1971 году японские математики T. Nomura и A. Fukuda предложили использовать его в качестве кодера и декодера двумерного ицклического кода [17]. В последующие годы появилась большая серия работ зарубежных специалистов, развивающая идеи Nomura и Fukuda. Особо следует упомянуть S. Sakata, опубликовавшего в 1977-1998 гг. 16 статей о многомерных рекуррентах и линейных регистрах сдвига. Тематика работ Sakata близка к криптографической; большое внимание уделяется обобщению известного алгоритма Берлекэмпа-Месси (см., например, [18]).

В России теория многомерных рекуррентных последовательностей и многомерных линейных (полилинейных) регистров сдвига развивается с середины 90-х гг. Трудами А. С. Кузьмина, В. Л. Куракина, А. В. Михалева и А.А.Нечаева были обобщены многие понятия и результаты классической теории ЛРП (см., например, [15]). Интерес к полилинейному (и, в частности, к 2-линейному) регистру сдвига обусловлен, главным образом, его криптографическими приложениями. В 2003 году в работе [10] А. А. Нечаев предложил автоматную модель полилинейного регистра сдвига, позволяющую генерировать на основе многомерной рекурренты одномерную псевдослучайную последовательность. Суть модели сводится к следующему:

1. Закон движения окна задается управляющей последовательностью $\delta$ : знак $\delta(i)$ определяет величину и направление «скачка» окна в $i$-й такт работы регистра.

2. Управляющая последовательность либо поступает на регистр извне (неавтономный случай), либо снимается с текущего заполнения регистра при помощи некоторой функиии самоуправления $\beta$ :

$$
\delta(i)=\beta(w(i)), \quad i=0,1,2, \ldots
$$

В последнем случае регистр называется самоуправляемым. 
3. Выходная одномерная последовательность $\gamma$ снимается с текущего заполнения регистра при помощи некоторой функции выхода $\psi$ :

$$
\gamma(i)=\psi(w(i)), \quad i=0,1,2, \ldots
$$

Автоматную модель естественно использовать в качестве центрального узла генератора псевдослучайных последовательностей. В работах [5-7] и [4] предпринята попытка исследования криптографических свойств генератора, построенного на основе 2-линейного регистра сдвига. Показано, что при подходящем выборе начального заполнения и функции самоуправления регистра ранг (линейная сложность) выходной последовательности оценивается снизу экспонентой от объема накопителя, а по своим частотным характеристикам эта последовательность близка к сбалансированной. Данная работа посвящена изучению периодических свойств автоматной модели 2-линейного регистра сдвига.

Переход к размерности $k>1$ приводит к существенному усложнению теории. Если в классическом случае рассматриваются период регистра и период рекурренты, то при $k=2$ возникает еще и период последовательности состояний автоматной модели. Обсудим эти понятия. Используемая далее терминология взята из работы [15].

Пусть идеал $I$ кольца $R_{2}=R\left[x_{0}, x_{1}\right]$ порожден многочленами $F\left(x_{0}\right)$ и $G\left(x_{1}\right)$ :

$$
I=\left(F\left(x_{0}\right), G\left(x_{1}\right)\right)_{R_{2}} .
$$

Вектор-периодом идеала $I$ называется всякий вектор $\left(t_{0}, t_{1}\right) \in \mathbb{N}_{0}^{2}$, для которого существует вектор $\left(l_{0}, l_{1}\right) \in \mathbb{N}_{0}^{2}$ со свойством

$$
x_{0}^{l_{0}} x_{1}^{l_{1}}\left(x_{0}^{t_{0}} x_{1}^{t_{1}}-1\right) \in I
$$

Вектор-периоды идеала $I$ порождают группу $(\mathfrak{P}(I),+)$. Мощность $T(I)$ факторгруппы $\mathbb{Z}^{2} / \mathfrak{P}(I)$ называется периодом идеала $I$ (или периодом соответствующего 2-ЛРС).

Описанное выше отображение $u: \mathbb{N}_{0}^{2} \rightarrow R$ называют 2-линейной рекуррентной последовательностью (2-ЛРП) с характеристическими многочленами $F$ и $G$. Множество всех 2-ЛРП с этими характеристическими многочленами обозначим через $L_{R}(F, G)$. Семейство рекуррент $L_{R}(F, G)$ является левым $R$-модулем. Равенства

$$
\left(x_{0} u\right)(i, j)=u(i+1, j), \quad\left(x_{1} u\right)(i, j)=u(i, j+1)
$$


наделяют это семейство структурой левого $R_{2}$-модуля. Вектор-периодом рекурренты $u$ называется всякий вектор $\left(t_{0}, t_{1}\right) \in \mathbb{N}_{0}^{2}$, для которого существует вектор $\left(l_{0}, l_{1}\right) \in \mathbb{N}_{0}^{2}$ со свойством

$$
x_{0}^{l_{0}} x_{1}^{l_{1}}\left(x_{0}^{t_{0}} x_{1}^{t_{1}}-1\right) u=0
$$

Вектор-периоды рекурренты $u$ порождают группу $(\mathfrak{P}(u),+)$. Мощность $T(u)$ фактор-группы $\mathbb{Z}^{2} / \mathfrak{P}(u)$ называется периодом рекурренты $u$.

При фиксированной функции самоуправления $\beta$ и фиксированном начальном состоянии ж(0) возникает последовательность

$$
w=(w(0), w(1), w(2), \ldots, w(i), \ldots)
$$

состояний самоуправляемого 2-ЛРС. Функция выхода $\psi$ позволяет построить выходную последовательность $\gamma($ см. (2)). Если $u[\mathfrak{F}]=w(0), T(w)-$ период последовательности ж, $T(\gamma)$ - период последовательности $\gamma$, то справедлива цепочка неравенств

$$
T(\gamma) \leq T(w) \leq T(u) \leq T(I)
$$

В работе [9] поставлен вопрос: при каких условиях на автоматную модель и начальное заполнение ш(0) все (или некоторые) из неравенств цеепочки (4) обрашчаются в равенства?

Пусть $R=G R\left(q^{n}, p^{n}\right)$ - кольц̧о Галуа мощности $q^{n}$ и характеристики $p^{n}$ (см., например, [11]). Унитарный многочлен $G(x) \in R[x]$ со свойством $G(0) \in R^{*}$ называется реверсивным многочленом. Реверсивный многочлен $G(x)$ степени $m \geq 1$ и периода $p^{n-1}\left(q^{m}-1\right)$ называется многочленом максимального периода (ММП) [15]. Настоящая работа отвечает на вопрос работы [9] в ситуации, когда $F(x)=G(x) \in R[x]-$ многочлен максимального периода, а функция $\psi$ линейна.

Введем ряд понятий.

1. В ситуации $F(x)=G(x)$ многочлен $F(x)$ будем называть характеристическим многочленом регистра $\mathfrak{A}$ (см. рис. 1).

2. Идеал $I=\left(F\left(x_{0}\right), F\left(x_{1}\right)\right)_{R_{2}}$ реверсивен [15], т. е. существует такая пара чисел $\left(t_{0}, t_{1}\right) \in \mathbb{N}^{2}$, что $\left.x_{0}^{t_{0}} \equiv x_{1}^{t_{1}} \equiv 1(\bmod I)\right)$. Для этой пары $\left(t_{0}, t_{1}\right)$ и для всякой 2-ЛРП $u \in L_{R}(F, F)$ верны равенства

$$
x_{0}^{t_{0}} u=u, \quad x_{1}^{t_{1}} u=u \text {. }
$$

Рассмотрим отношение « » на множестве $L_{R}(F, F)$ :

$$
u \sim u^{\prime} \Leftrightarrow \exists t_{0}, t_{1} \in \mathbb{N}: x_{0}^{t_{0}} u=u^{\prime}, \quad x_{1}^{t_{1}} u=u^{\prime} .
$$




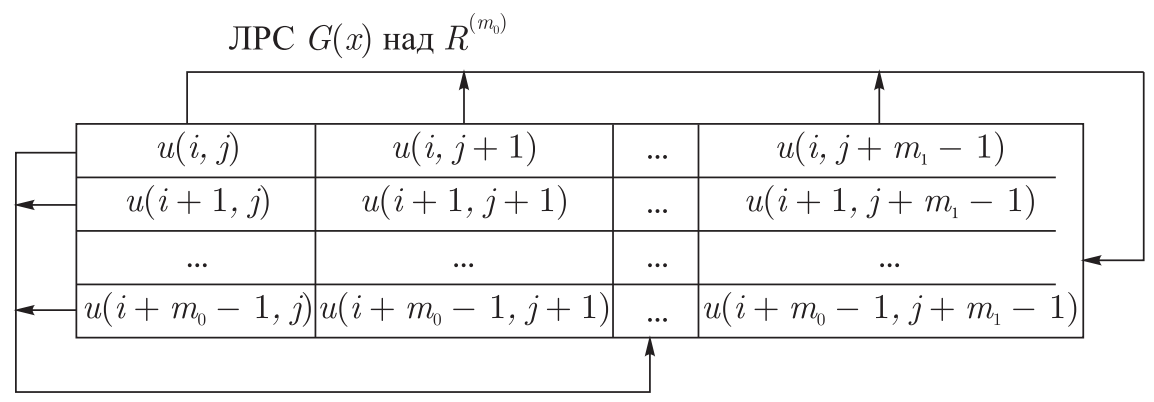

ЛРС $F(x)$ над $R^{m_{1}}$

Рис. 1. 2-линейный регистр сдвига $\mathfrak{A}$

Согласно (5), это отношение рефлексивно. Оно транзитивно, и, как легко заметить, симметрично. Таким образом, отношение « » есть отношение эквивалентности. Следуя [15], назовем класс эквивалентности

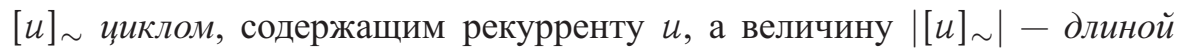
этого цикла. Очевидно, что длина цикла, содержащего рекурренту $u$, совпадает с периодом $T(u)$ этой рекурренты.

3. Положим

$$
\Omega_{m}=R_{m, m}, \quad \Omega=\bigcup_{m=1}^{\infty} \Omega_{m} .
$$

Пусть предикат $A$ задан на множестве $\Omega$. Будем говорить, что условие $A$ выполняется почти для всех $z \in \Omega$, если существует такая функция $\alpha: \mathbb{N} \rightarrow \mathbb{R}$, что

$$
\alpha(m)=o(1), \quad m \rightarrow \infty,
$$

и для любого $m \geq 1$ доля матриц $z \in \Omega_{m}$, для которых $A$ не выполняется, не превосходит $\alpha(m)$.

4. Выберем и зафиксируем последовательность многочленов максимального периода над кольцом $R$ :

$$
F_{1}(x), F_{2}(x), \ldots, F_{m}(x), \ldots,
$$

где $\operatorname{deg} F_{m}(x)=m, m=1,2, \ldots$ Функцию самоуправления $\beta: \Omega \rightarrow$ $\rightarrow\{0,1\}$ назовем оптимальной для последовательности (7), если почти для всех

$$
T(w)=T(u),
$$


где рекуррента $u \in L_{R}\left(F_{m}, F_{m}\right)$ определена равенством $u[\mathfrak{F}]=w(0)$, а w - последовательность (3) состояний 2-ЛРС с характеристическим многочленом $F_{m}(x)$ и функцией самоуправления $\beta, m=1,2, \ldots$

5. Всюду в этой работе предполагается, что последовательность (7) фиксирована. Поэтому далее вместо термина «функция, оптимальная для последовательности многочленов (7)» будем употреблять термин «оптимальная функция».

Опишем полученные результаты.

1. При некоторых ограничениях на кольцо $R$, многочлен максимального периода $F(x) \in R[x]$ и рекурренту $u \in L_{R}(F, F)$ вычислен период $T(u)$ (теорема 3 ). В случае, когда $R$ - поле, а $F(x)$ - произвольный многочлен максимального периода, найден цикловой тип семейства $L_{R}(F, F)$, т. е. для всякого $t \geq 1$ указано число рекуррент $u \in L_{R}(F, F)$, принадлежащих циклам длины $t$ (следствие 2).

2. В случае, когда $R$ - поле, построен класс $B_{1}$ оптимальных функций самоуправления, вообще говоря, нелинейных (утверждение 4). В случае $R=\mathbb{Z}_{2}$ показано, что для всякой оптимальной функции $\beta$ существует функция $\beta^{\prime} \in B_{1}$, совпадающая с $\beta$ почти во всех точках $z \in \Omega$ (следствие 4).

3. В случае, когда $R$ - поле, для произвольной функции самоуправления $\beta \in B_{1}$ и всякой линейной функции выхода $\psi$ исследована связь между периодом $T(w)$ последовательности состояний ш и периодом $T(\gamma)$ выходной последовательности $\gamma($ теорема 6). Показано, что «почти все» линейные функции выхода сохраняют период последовательности состояний (следствие 5).

\section{2. О цикловом типе семейства $L_{R}(F, F)$}

Пусть $r, n \in \mathbb{N}, p$ - простое число, $q=p^{r}, R=G R\left(q^{n}, p^{n}\right)$ - кольцо Галуа мощности $q^{n}$ и характеристики $p^{n}, F(x) \in R[x]-$ многочлен максимального периода степени $m \geq 1$. Вычислим величину

$$
L(F)=\max \left\{T(u) \mid u \in L_{R}(F, F)\right\}
$$

- максимум длин циклов семейства $L_{R}(F, F)$.

Пусть $I=\left(F\left(x_{0}\right), F\left(x_{1}\right)\right)_{R_{2}} \triangleleft R_{2}$. Очевидно, $L(F) \leq T(I)$. Согласно [8, следствие 2], имеем:

$$
T(I)=\frac{T(F)^{2}}{|\operatorname{Mult}(F)|},
$$


где

$$
\operatorname{Mult}(F)=\left\{a \in R^{*}: F(x) \mid x^{t}-a \text { при некотором } t \geq 0\right\}
$$

- группа мультипликаторов многочлена $F(x)$. Изучим свойства группы мультипликаторов.

В расширении $Q=G R\left(q^{m n}, p^{n}\right)$ кольца $R$ содержится корень $\theta$ многочлена $F(x)$, причем $\theta \in Q^{*}$. Пусть $\tau=q^{m}-1$. Группа $Q^{*}$ является прямым произведением своих подгрупп

$$
\Gamma(Q)=\left\{x \in Q \mid x^{\tau}=1\right\} \quad \text { и } \quad 1+p Q,
$$

причем $\Gamma(Q)$ - циклическая группа порядка $\tau$. Пусть $\theta=\theta_{0} \cdot \theta_{1}$, где $\theta_{0} \in \Gamma(Q)$ и $\theta_{1} \in 1+p Q$. В случае, когда $R=\mathbb{Z}_{4}$ или $p>2$, группа $1+p Q$ является прямым произведением группы $1+p R$ и некоторой своей подгруппы $G$ (см. [16, глава 16]):

$$
1+p Q=(1+p R) \dot{\times} G
$$

причем

$$
\exp \{1+p R\}=p^{n-1}, \quad \exp G \mid p^{n-1}
$$

(см., например, [11, теорема 3.1]). Элемент $\theta_{1}$ однозначно представляется в виде

$$
\theta_{1}=\theta_{1,1} \cdot \theta_{1,2}
$$

где $\theta_{1,1} \in 1+p R, \theta_{1,2} \in G$. Определим числа $k_{1}, k_{2} \in\{0,1, \ldots, n-1\}$ равенствами

$$
\text { ord } \theta_{1,1}=p^{k_{1}}, \quad \text { ord } \theta_{1,2}=p^{k_{2}} \text {. }
$$

Утверждение 1 ([3, утверждение 4, пункт 4]). Если

$$
R-\text { поле, или } R=\mathbb{Z}_{4} \text {, или } p>2,
$$

mo $|\operatorname{Mult}(F)|=(q-1) p^{\nu}$, где

$$
\nu=\nu(F)=\left\{\begin{array}{lll}
0, & \text { если } & k_{1}<n-1, \\
n-1-k_{2}, & \text { если } & k_{1}=n-1 .
\end{array}\right.
$$

Из утверждения 1 вытекает следующий факт.

Следствие 1. При условии (11) над кольияом $R$ сущуествует многочлен максимального периода $F(x)$, для которого $|\operatorname{Mult}(F)|=(q-1) p^{n-1}$. 
Доказательство. Группа $\operatorname{Aut}(Q / R)$ автоморфизмов кольца $Q$, оставляющих элементы кольца $R$ неподвижными, является циклической. Пусть $\varphi-$ ее циклический образующий:

$$
\operatorname{Aut}(Q / R)=\langle\varphi\rangle .
$$

В группе $\Gamma(Q)$ есть циклический образующий $\theta_{0}$. Ввиду $(10)$ и свойств конечных абелевых групп (см., например, [1, том 1, глава 11, утверждение 2]) существует элемент $\theta_{1} \in 1+p R$ со свойством ord $\theta_{1}=p^{n-1}$. Положим $\theta=\theta_{0} \cdot \theta_{1}$. Согласно утверждению 1 , многочлен

$$
F(x)=(x-\theta)(x-\varphi(\theta)) \cdots\left(x-\varphi^{m-1}(\theta)\right) \in R[x]
$$

является искомым. \

Многочлены максимального периода $F(x) \in R[x]$, описываемые следствием 1 , будем называть особыми. Очевидно, что над полем всякий многочлен максимального периода является особым. Всюду далее в этом параграфе будем считать, что выполнено условие (11) и $F(x)$ - особый многочлен максимального периода (особый ММП).

Из равенства (9) и следствия 1 вытекает, что при условии (11) для всякого особого ММП $F(x) \in R[x]$ верно неравенство

$$
L(F) \leq T(I)=p^{n-1} \frac{\left(q^{m}-1\right)^{2}}{q-1} .
$$

Покажем, что на самом деле при указанных условиях справедливо равенство

$$
L(F)=p^{n-1} \frac{\left(q^{m}-1\right)^{2}}{q-1} .
$$

Для этого опишем способ построения таких рекуррент $u \in L_{R}(F, F)$, что период $T(u)$ совпадает с правой частью неравенства (12). Такие рекурренты удобно строить, опираясь на результаты работы [4] о периодических свойствах 2-ЛРС.

Сначала формально опишем неавтономный 2-ЛРС. Пусть $S(F)-$ сопровождающая матрица многочлена $F(x)$ (см., например, [1]):

$$
S(F)=\left(\begin{array}{ccccc}
0 & \ldots & 0 & 0 & f_{0} \\
1 & \ldots & 0 & 0 & f_{1} \\
\vdots & \ddots & \vdots & \vdots & \vdots \\
0 & \ldots & 1 & 0 & f_{m-2} \\
0 & \ldots & 0 & 1 & f_{m-1}
\end{array}\right),
$$


где коэффициенты $f_{0}, f_{1}, \ldots, f_{m-1} \in R$ определены равенством

$$
F(x)=x^{m}-f_{m-1} x^{m-1}-f_{m-2} x^{m-2}-\cdots-f_{1} x-f_{0} .
$$

Обозначим через $e_{i, j}$ матричную единицу, т. е. $m \times m$-матрицу, в которой на пересечении $i$-й строки и $j$-го столбца стоит 1 , а на остальных позициях -0 (нумерация строк и столбцов ведется с нуля). Рассмотрим $\boldsymbol{e}^{\downarrow}-$ записанный столбцом базис модуля ${ }_{R} \Omega_{m}$, состоящий из матричных единиц:

$$
\boldsymbol{e}^{\downarrow}=\left(e_{0,0}, \ldots, e_{0, m-1}, e_{1,0}, \ldots, e_{1, m-1}, \ldots\right)^{T}
$$

и автоморфизмы $\varphi_{x} \in \operatorname{Aut}\left(\Omega_{m}\right), x=0,1$, определенные равенством

$$
\forall z \in \Omega_{m}: \varphi_{x}(z)=\vec{z} \cdot\left(S(F)^{1-x} \otimes S(F)^{x}\right) \cdot \boldsymbol{e}^{\downarrow},
$$

где $\vec{z}-$ строка координат матрицы $z$ в базисе $\boldsymbol{e}^{\downarrow}, \otimes-$ операция тензорного (кронекерова) произведения матриц (см., наример, [12]). Неавтономный автомат

$$
\mathfrak{A}=\left(\{0,1\}, \Omega_{m}, R,\left\{\varphi_{0}, \varphi_{1}\right\}, \psi\right)
$$

со входным алфавитом $\{0,1\}$, множеством состояний $\Omega_{m}$, выходным алфавитом $R$, частичными функциями перехода $\varphi_{0}$ и $\varphi_{1}$ и функцией выхода $\psi: \Omega_{m} \rightarrow R$ называется неавтономнылм 2-линейным регистром сдвига (неавтономным 2-ЛРС).

Пусть $\bar{R}=R / p R, \bar{F}$ - образ многочлена $F$ под действием естественного эпиморфизма $R \rightarrow \bar{R}$. Рассмотрим последовательное соединение фильтрующзего генератора (см., например, [14]) и неавтономного 2-линейного регистра сдвига $\mathfrak{A}$.

Здесь $v \in L_{\bar{R}}(\bar{F})-$ ЛРП максимального периода (ЛРП МП) $\tau$ над полем $\bar{R}$, а

$$
f: \bar{R}^{m} \rightarrow\{0,1\}
$$

- функция со свойством $f(0,0, \ldots, 0)=0$, вес

$$
\|f\|=\left|\left\{\left(a_{0}, a_{1}, \ldots, a_{m-1}\right) \in \bar{R}^{m}: f\left(a_{0}, a_{1}, \ldots, a_{m-1}\right)=1\right\}\right|
$$

которой взаимно прост с $\tau$. Тогда $T(\delta)=\tau$.

Рассмотрим последовательность состояний (3), вырабатываемую автоматом $\mathfrak{A}$ в указанном последовательном соединении:

$$
w=(w(0), w(1), w(2), \ldots, w(i), \ldots) .
$$

Свойства последовательности ш изучены в работе [4]. Перечислим основные результаты этой работы. 
Реверсивный многочлен $G(x) \in R[x]$ называется многочленом Галуа (см., например, [3]), если его образ $\bar{G}[x]$ под действием естественного эпиморфизма $R \rightarrow \bar{R}$ неприводим над $\bar{R}$, и примарным многочленом [11], если его образ под действием указанного эпиморфизма есть неприводимый многочлен в натуральной степени. Согласно [2] каноническим разложением реверсивного многочлена над кольцом $R$ называется его разложение в произведение попарно взаимно простых примарных сомножителей.

Пусть $k_{f}=\|f\|$, и автоморфизм $\sigma$ модуля ${ }_{R} \Omega_{m}$ определен равенством

$$
\sigma=\varphi_{0}^{-p^{n-1} k_{f}} \varphi_{1}^{p^{n-1} k_{f}} .
$$

Следующая теорема доказана в работе [4] для случая $p=2$, однако предложенное там доказательство дословно переносится на случай $p>2$ :

Теорема 1. Характеристический многочлен $\chi_{\sigma}(x)$ автоморфизма $\sigma$ имеет каноническое разложение:

$$
\chi_{\sigma}(x)=G_{0}(x) G_{1}(x) \ldots G_{m-1}(x),
$$

где $G_{0}(x)=(x-1)^{m}$, a $G_{1}(x), G_{2}(x), \ldots, G_{m-1}(x)$ - попарно взаимно простые многочлены Галуа степени $m$, причем $\bar{G}_{s}(x)$ является минимальным многочленом элемента $\bar{\theta}^{-p^{n-1} k_{f}\left(q^{s}-1\right)} \in \bar{Q}$ над полем $\bar{R}, s=1,2, \ldots, m-1$.

Разложение (17) индуцирует однозначное разложение начального состояния ๗(0) $\in \Omega_{m}$ :

$$
w(0)=z_{0}+z_{1}+\ldots+z_{m-1},
$$

где $z_{s} \in \operatorname{Ker} G_{s}(\sigma), s=0,1, \ldots, m-1$. Пусть $\varepsilon_{s}-$ индикатор неравенства $z_{s} \neq$ $\neq 0, s=0,1, \ldots, m-1$. Следуя [1], для произвольных чисел $a_{1}, a_{2}, \ldots, a_{k} \in$ $\in \mathbb{Z}$ обозначим через $\left(a_{1}, a_{2}, \ldots, a_{k}\right)$ их наибольший общий делитель, а через $\left[a_{1}, a_{2}, \ldots, a_{k}\right]-$ их наименьшее общее кратное. Сформулированное ниже утверждение доказано в [4, теорема 8] для случая $p=2$. На случай $p>2$ доказательство переносится дословно.

Теорема 2. Пусть в разложении (18) $\bar{z}_{0} \neq 0 u$

$$
\forall s \in\{1,2, \ldots, m-1\}: \tau_{s}=\tau /\left(q^{(m, s)}-1\right) .
$$

Тогда

$$
T(w)=p^{n-1} \tau \cdot\left[\tau_{1}^{\varepsilon_{1}}, \tau_{2}^{\varepsilon_{2}}, \ldots, \tau_{m-1}^{\varepsilon_{m-1}}\right] \leq p^{n-1} \frac{\tau^{2}}{q-1} .
$$


В частности, равенство

$$
T(w)=p^{n-1} \frac{\tau^{2}}{q-1}
$$

имеет место тогда и только тогда, когда

$$
\left(\varepsilon_{1}, 2 \varepsilon_{2}, 3 \varepsilon_{3}, \ldots,(m-1) \varepsilon_{m-1}, m\right)=1 .
$$

Если $\bar{z}_{0} \neq 0$, то, согласно [4, утверждение 3], существует такая ЛРП МП $v \in L_{\bar{R}}(\bar{F})$, что

$$
\bar{z}_{0}=\sum_{i=0}^{m-1} \sum_{j=0}^{m-1} v(i+j) \cdot e_{i, j} .
$$

Будем говорить, что ЛРП $v$ ассоииирована с начальным заполнением ж(0) (см. разложение (18)). Тогда для всякого $i \geq 0$ ЛРП $x^{i}$ च ассоциирована с состоянием ж(i). Положим

$$
\forall z \in \Omega_{m}: \quad \beta(z)=f(a(0), a(1), \ldots, a(m-1)),
$$

где $a \in L_{\bar{R}}(\bar{F})-$ ЛРП, ассоциированная с $z$. Тогда

$$
\forall i \geq 0: \beta(w(i))=f(v(i), v(i+1), \ldots, v(i+m-1))=\delta(i),
$$

где $\delta$ - промежуточная последовательность в последовательном соединении, изображенном на рисунке 2 .

Используя функцию $\beta$ вида (21) в качестве функции самоуправления, построим самоуправляемый 2-линейный регистр сдвига $\mathfrak{A}^{\beta}$ (см. [10]):

$$
\mathfrak{A}^{\beta}=\left(\Omega_{m}, R, h_{\beta}, \psi\right),
$$

где $\Omega_{m}-$ множество состояний, $R-$ выходной алфавит, $\psi: \Omega_{m} \rightarrow R-$ функция выхода, а $h_{\beta}-$ функция перехода:

$$
\forall z \in \Omega_{m}: \quad h_{\beta}(z)=\varphi_{\beta(z)}(z) .
$$

Если последовательности ш справедлива теорема 2. С другой стороны, в силу (4) для 2-ЛРП $u \in L_{R}(F, F)$, определенной равенством $u[\mathfrak{F}]=w(0)$, верна цепочка неравенств

$$
T(w) \leq T(u) \leq T(I) .
$$




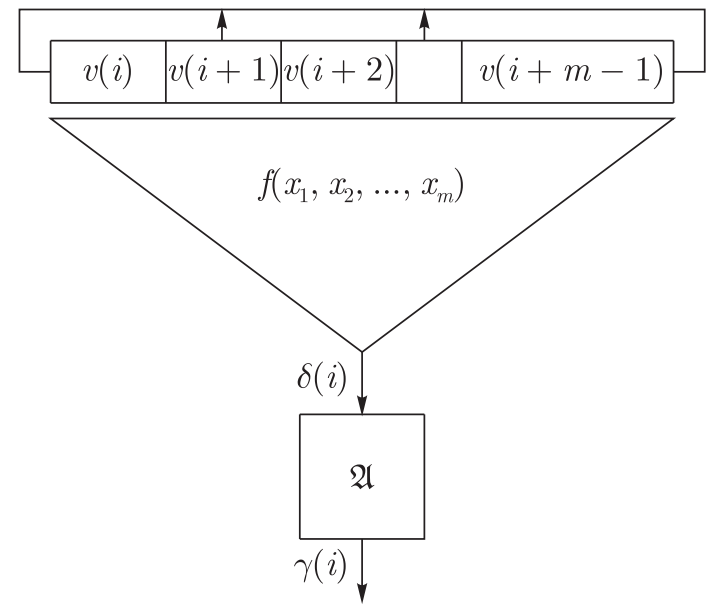

Рис. 2. Последовательное соединение 1-ЛРС и 2-ЛРС

Таким образом, при условии (11) для всякого особого ММП $F(x)$ и всякой рекурренты $u \in L_{R}(F, F)$, начальный вектор $u[\mathfrak{F}]=w(0)$ которой удовлетворяет условиям $\bar{z}_{0} \neq 0$ и (19), имеем

$$
p^{n-1} \frac{\tau^{2}}{q-1}=T(w) \leq T(u) \leq T(I)=p^{n-1} \frac{\tau^{2}}{q-1} .
$$

Напомним (см. параграф 1), что цикл определяется как класс попарно эквивалентных рекуррент. Поэтому можно говорить о рекуррентах, принадлежащих данному циклу.

Теорема 3. При условии (11) для всякого особого ММП $F(x)$ количество $N(F)$ рекуррент $u \in L_{R}(F, F)$ со свойством

$$
T(u)=T(I)=p^{n-1} \frac{\tau^{2}}{q-1}
$$

удовлетворяет неравенству

$$
N(F) \geq\left(q^{m}-1\right) \sum_{d \mid m} \mu(m / d) \cdot q^{m(n d-1)},
$$

где $\mu$ - функиия Мебиуса [12]. В частности, верно равенство (13), и доля рекуррент $и \in L_{R}(F, F)$, принадлежащих ииклам максимальной длины $T(I)$, есть $1+o(1)$ nри $m \rightarrow \infty$. 
Доказательство. Пусть $N_{1}$ - правая часть неравенства (24). Согласно $[4$, следствие 10$]$, величина $N_{1}$ равна количеству состояний $(0) \in \Omega_{m}$, удовлетворяющих условиям $\bar{z}_{0} \neq 0$ и (19). Осталось заметить, что

$$
N_{1}=q^{m^{2} n}(1+o(1))=|R|^{m^{2}}(1+o(1)), \quad m \rightarrow \infty . \triangleright
$$

Теорема 3 описывает почти весь цикловой тип семейства $L_{R}(F, F)$. В случае, когда $R$ - конечное поле, цикловой тип можно описать полностью.

Всюду далее в этом параграфе $n=1$, т.е. $R=G F(q)$. Пусть $u \in$ $\in L_{R}(F, F), H=\tau \mathbb{Z} \times \tau \mathbb{Z}$ и

$$
\overline{\mathfrak{P}}(u)=\mathfrak{P}(u) \cap(\{0,1, \ldots, \tau-1\} \times\{0,1, \ldots, \tau-1\}) .
$$

Так как $\mathbb{Z}^{2} \geq \mathfrak{P}(u) \geq H$, по второй теореме об изоморфизме групп [1, том 1 , с. 292, теорема 29] имеем

$$
T(u)=\left|\mathbb{Z}^{2} / \mathfrak{P}(u)\right|=\left|\left(\mathbb{Z}^{2} / H\right) /(\mathfrak{P}(u) / H)\right|=\tau^{2} \cdot|\overline{\mathfrak{P}}(u)|^{-1} .
$$

Пусть $u[\mathfrak{F}]=w(0) \neq 0$. Удалив из разложения (18) нулевые слагаемые, получим

$$
w(0)=z_{i_{1}}+z_{i_{2}}+\cdots+z_{i_{t}},
$$

где $0 \leq i_{1}<i_{2}<\cdots<i_{t} \leq m-1$. Если рекуррента $u_{i_{s}} \in L_{R}(F, F)$ определена равенством $u_{i_{s}}[\mathfrak{F}]=z_{i_{s}}, s=1,2, \ldots, t$, то имеет место следующее однозначное разложение рекурренты $u$ :

$$
u=u_{i_{1}}+u_{i_{2}}+\cdots+u_{i_{t}},
$$

где $u_{i}[\mathfrak{F}] \in \operatorname{Ker} G_{i}(\sigma) \backslash\{0\}, i \in\left\{i_{1}, i_{2}, \ldots, i_{t}\right\}$. Отсюда

$$
\mathfrak{P}(u)=\bigcap_{s=1}^{t} \mathfrak{P}\left(u_{i_{s}}\right),
$$

и, следовательно,

$$
|\overline{\mathfrak{P}}(u)|=\left|\bigcap_{s=1}^{t} \overline{\mathfrak{P}}\left(u_{i_{s}}\right)\right| .
$$

Вычислим правую часть равенства (27). 
Лемма 1. Во введенных обозначениях справедливо равенство

$$
\left|\bigcap_{s=1}^{t} \overline{\mathfrak{P}}\left(u_{i_{s}}\right)\right|=q^{\left(m, i_{2}-i_{1}, i_{3}-i_{1}, \ldots, i_{t}-i_{1}\right)}-1 .
$$

Доказательство. Всякая рекуррента $u \in L_{R}(F, F)$ представляет собой бесконечную вправо и вниз таблицу (см. параграф 1.). Будем нумеровать строки этой таблицы, начиная с нуля. Пусть $s \in\{1,2, \ldots, t\}$. Согласно [4, утверждение 3] существует такая ненулевая рекуррента $v_{s} \in L_{R}(F)$, что для всякого $j \geq 0 j$-я строка рекурренты $u_{i_{s}}$ имеет вид

$$
\left(x^{j q^{i_{s}}} v_{s}\right)(0,1, \ldots, m-1) .
$$

Поэтому включение $\mathbf{t} \in \overline{\mathfrak{P}}\left(u_{i_{s}}\right)$ равносильно тому, что первая координата вектора $\mathbf{t}$ равна $j$, а вторая координата есть $\tau-j q^{i_{s}}$ для подходящего $j \in\{0,1, \ldots, \tau-1\}$ (операции во второй координате осуществляются по модулю $\tau$ ). Поэтому левая часть равенства (28) равна числу решений системы сравнений

$$
\left\{\begin{array}{c}
j q^{i_{1}} \equiv j q^{i_{2}}(\bmod \tau) \\
j q^{i_{1}} \equiv j q^{i_{3}}(\bmod \tau) \\
\vdots \\
j q^{i_{1}} \equiv j q^{i_{t}}(\bmod \tau)
\end{array}\right.
$$

относительно $j \in\{0,1, \ldots, \tau-1\}$. Множество решений $l$-го сравнения этой системы, $l=1,2, \ldots, t-1$, имеет вид

$$
\frac{\tau}{q^{\left(m, i_{l+1}-i_{1}\right)}-1} \cdot i, \quad i=0,1,2, \ldots
$$

Поэтому количество решений системы (29) равно величине

$$
\tau \times\left\{\left[\frac{\tau}{q^{\left(m, i_{2}-i_{1}\right)}-1}, \frac{\tau}{q^{\left(m, i_{3}-i_{1}\right)}-1}, \ldots, \frac{\tau}{q^{\left(m, i_{t}-i_{1}\right)}-1}\right]\right\}^{-1},
$$

совпадающей с правой частью равенства (28). $\triangleright$

Из равенств (25), (27) и (28) вытекает следующая теорема.

Теорема 4. Пусть $R=G F(q), F(x) \in R[x]-$ многочлен максимального периода, $u \in L_{R}(F, F)$. Если $u=0$, то $T(u)=1$. Если в разложении (26) $t=1$, то $T(u)=\tau$. Если в разложении (26) $t \geq 2$, то

$$
T(u)=\frac{\tau^{2}}{q^{d}-1},
$$

где $d=\left(m, i_{2}-i_{1}, i_{3}-i_{1}, \ldots, i_{t}-i_{1}\right)$. 
Следствие 2. В условиях теоремь 4 в семействе $L_{R}(F, F)$ одна (нулевая) рекуррента имеет период $1, m \tau$ рекуррент имеют период $\tau$, и для любого $d \mid m$ такого, что $1 \leq d<m$, число рекуррент, имеющих период $\tau^{2} /\left(q^{d}-1\right)$, равно

$$
\sum_{\substack { t=2 \\
\begin{subarray}{c}{1 \leq j_{2}<j_{3}<\cdots<j_{t} \leq m-1 \\
\left(j_{2}, j_{3}, \ldots, j_{t}, m\right)=d{ t = 2 \\
\begin{subarray} { c } { 1 \leq j _ { 2 } < j _ { 3 } < \cdots < j _ { t } \leq m - 1 \\
( j _ { 2 } , j _ { 3 } , \ldots , j _ { t } , m ) = d } }\end{subarray}}\left(m-j_{t}\right) \tau^{t} .
$$

Доказательство. Равенство $T(0)=1$ очевидно. По теореме 4 условия $T(u)=\tau$ и $t=1$ эквивалентны (см. разложение (26)). Найдем число рекуррент $u \in L_{R}(F, F)$ со свойством $t=1$. Значение $i_{1}$ из множества $\{0,1, \ldots, m-1\}$ выбирается $m$ способами. Ненулевая рекуррента $u_{i_{1}} \in$ $L_{R}(F, F)$ со свойством $u_{i_{1}}[\mathfrak{F}] \in \operatorname{Ker} G_{i_{1}}(\sigma)$ выбирается

$$
\left|\operatorname{Ker} G_{i_{1}}(\sigma)\right|-1=|R|^{\operatorname{deg} G_{i_{1}}}-1=q^{m}-1=\tau
$$

способами. Поэтому количество рекуррент $u \in L_{R}(F, F)$ со свойством $T(u)=\tau$ равно $m \tau$.

Пусть теперь $d \mid m$ и $1 \leq d<m$. Вычислим количество рекуррент $u \in L_{R}(F, F)$ со свойством (30). По теореме 4 для таких рекуррент $t \geq 2$. Положим $j_{s}=i_{s}-i_{1}, s=2,3, \ldots, t$. Для того чтобы построить рекурренту $u$ со свойством (30), необходимо сначала выбрать набор $\left(i_{1}, i_{2}, \ldots, i_{t}\right)$ со свойствами $0 \leq i_{1}<i_{2}<\cdots<i_{t} \leq m-1$ и $\left(j_{2}, j_{3}, \ldots, j_{t}, m\right)=d$, а затем выбрать рекурренты $u_{i_{s}} \in L_{R}(F, F)$ со свойством $u_{i_{s}}[\mathfrak{F}] \in \operatorname{Ker} G_{i_{s}}(\sigma), s=1,2, \ldots, t$. Поэтому в семействе $L_{R}(F, F)$ существует

$$
\sum_{\substack{t=2 \\ 1 \leq j_{2}<j_{3}<\cdots<j_{t} \leq m-1 \\\left(j_{2}, j_{3}, \ldots, j_{t}, m\right)=d}} \sum_{\substack{i_{1}=0 \\ m-j_{t}-1}}^{m} \tau^{t}
$$

рекуррент $u$ со свойством (30). Величина (32), очевидно, равна величине (31). Формула (31) является точной, однако вычисления по ней довольно трудны. В связи с этим может быть полезна следующая оценка.

Следствие 3. В условиях теоремы 4 в семействе $L_{R}(F, F)$ существует не менее

$$
\left(q^{m}-1\right) \sum_{d_{1} \mid \frac{m}{d}} \mu\left(\frac{m}{d d_{1}}\right) q^{m\left(d_{1}-1\right)}
$$

рекуррент и со свойством (30), где $\mu-$ функция Мебиуса. 
Доказательство. Покажем, что величина (33) равна количеству $M_{d}(F)$ рекуррент $u \in L_{R}(F, F)$ со свойством (30), у которых $i_{1}=0$.

Пусть $a \geq 2,1 \leq s<a$. Обозначим через $\varphi^{[s]}(a)$ количество наборов $\left(l_{1}, l_{2}, \ldots, l_{s}\right)$ таких, что $1 \leq l_{1}<l_{2}<\cdots<l_{s} \leq a-1$ и $\left(l_{1}, l_{2}, \ldots, l_{s}, a\right)=1$. Согласно [6, лемма 20], верно равенство

$$
\varphi^{[s]}(a)=\sum_{d_{1} \mid a} \mu\left(\frac{a}{d_{1}}\right)\left(\begin{array}{c}
d_{1}-1 \\
s
\end{array}\right) .
$$

Между наборами $1 \leq i_{2}<i_{3}<\cdots<i_{t}<m$ со свойством $\left(i_{2}, i_{3}, \ldots, i_{t}, m\right)=d$ и наборами $1 \leq i_{2}^{\prime}<i_{3}^{\prime}<\cdots<i_{t}^{\prime}<\frac{m}{d}$ со свойством $\left(i_{2}^{\prime}, i_{3}^{\prime}, \ldots, i_{t}^{\prime}, \frac{m}{d}\right)=1$ существует взаимнооднозначное соответствие. Количество и тех, и других наборов равно $\varphi^{[t-1]}(m / d)$.

При фиксированном $t \in\{2,3, \ldots, m\}$ количество рекуррент $u \in$ $\in L_{R}(F, F)$, принадлежащих циклам длины $\tau^{2} /\left(q^{d}-1\right)$, имеющих в разложении (26) $t$ слагаемых и обладающих свойством $i_{1}=0$, выражается формулой

$$
(|Q|-1)(|Q|-1)^{t-1} \varphi^{[t-1]}(m / d)=\left(q^{m}-1\right)\left(q^{m}-1\right)^{t-1} \varphi^{[t-1]}(m / d) .
$$

Суммируя эту величину по $t$, получим величину $M_{d}(F)$ :

$$
M_{d}(F)=\left(q^{m}-1\right) \sum_{t=2}^{m}\left(q^{m}-1\right)^{t-1} \varphi^{[t-1]}(m / d) .
$$

Сделаем замену переменной $s=t-1$ и применим формулу (34):

$$
\begin{gathered}
M_{d}(F)=\left(q^{m}-1\right) \sum_{s=1}^{m-1}\left(q^{m}-1\right)^{s} \varphi^{[s]}(m / d)= \\
=\left(q^{m}-1\right) \sum_{d_{1} \mid \frac{m}{d}} \mu\left(\frac{m}{d d_{1}}\right) \sum_{s=1}^{m-1}\left(q^{m}-1\right)^{s}\left(\begin{array}{c}
d_{1}-1 \\
s
\end{array}\right)= \\
=\left(q^{m}-1\right) \sum_{d_{1} \mid \frac{m}{d}} \mu\left(\frac{m}{d d_{1}}\right) \sum_{s=0}^{d_{1}-1}\left(q^{m}-1\right)^{s}\left(\begin{array}{c}
d_{1}-1 \\
s
\end{array}\right)= \\
=\left(q^{m}-1\right) \sum_{d_{1} \mid \frac{m}{d}} \mu\left(\frac{m}{d d_{1}}\right) q^{m\left(d_{1}-1\right)} .
\end{gathered}
$$

Формула (33) доказана. $\triangleright$

Следствие 3 уточняет теорему 3 в случае $n=1$. 


\section{3. Об оптимальном самоуправлении}

В этом и следующем параграфах $n=1$, т. е. $R=G F(q)$ - конечное поле. Тогда условие (11) выполнено, и для любого $m \geq 1$ многочлен $F_{m}(x)$ из ряда (7) является особым. Для краткости многочлен $F_{m}(x)$ будем обозначать через $F(x)$. Изучим оптимальные функции самоуправления $\beta: \Omega \rightarrow\{0,1\}$.

Рассмотрим автоморфизм $\rho=\varphi_{0}^{-1} \cdot \varphi_{1}$ пространства ${ }_{R} \Omega_{m}$. В обозначениях параграфа 2 автоморфизм $\rho-$ это автоморфизм $\sigma$ при $k_{f}=1$. Из теоремы 1 вытекает следующее утверждение.

Утверждение 2. Характеристический многочлен $\chi_{\rho}(x)$ автоморфизма $\rho$ имеет каноническое разложение:

$$
\chi_{\rho}(x)=H_{0}(x) H_{1}(x) \ldots H_{m-1}(x),
$$

где $H_{0}(x)=(x-1)^{m}$, а $H_{1}(x), H_{2}(x), \ldots, H_{m-1}(x)$ - попарно разные неприводимые над $R$ многочлены степени $m$, причем $H_{s}(x)$ является минимальным многочленом элемента $\theta^{-\left(q^{s}-1\right)} \in Q$ над полем $R, s=1,2, \ldots, m-1$.

Из [4, утверждение 3] следует, что для всякого $k_{f} \in\{1,2, \ldots, \tau-1\}$, взаимно простого с $\tau$, и для любого $s \in\{0,1, \ldots, m-1\}$ верно равенство

$$
\operatorname{Ker} G_{s}(\sigma)=\operatorname{Ker} H_{s}(\rho) .
$$

Это означает, что однозначное разложение

$$
w(0)=z_{0}+z_{1}+\cdots+z_{m-1},
$$

где $z_{s} \in \operatorname{Ker} H_{s}(\rho), s=0,1, \ldots, m-1$, совпадает с разложением (18).

Пусть $\varepsilon_{s}$ - индикатор того, что в разложении (35) $z_{s} \neq 0, s=$ $=0,1, \ldots, m-1$. Поскольку разложение (35) совпадает с разложением (18), введенное таким образом $\varepsilon_{s}$ совпадает с $\varepsilon_{s}$ из формулировки теоремы 2 . Сопоставим разложению (35) вектор

$$
\text { Type }(0)=\left(\varepsilon_{0}, \varepsilon_{1}, \ldots, \varepsilon_{m-1}\right) \in\{0,1\}^{m},
$$

который будет называться типом состояния ж(0). Типом рекурренты $u \in$ $\in L_{R}(F, F)$ назовем вектор Туре $u[\mathfrak{F}]$ (обозначение: Туре $u$ ). Подпространства $\operatorname{Ker} H_{s}(\rho)$ инвариантны относительно автоморфизмов $\varphi_{0}$ и $\varphi_{1}, s=$ $=0,1, \ldots, m-1$. Поэтому типы рекуррент, принадлежащих одному циклу, равны. Введем понятие типа ичикла: если $C \subset L_{R}(F, F)$ - цикл, содержащий рекурренту $u$, то Туре $C=$ Туре $u$. 
Согласно [5, теорема 2], разложение (35) и тип Туре $z$ данного состояния $z \in \Omega_{m}$ эффективно вычисляются за $O\left(m^{4}\right)$ операций сложения и умножения в поле $R$.

О функции $f: R^{m} \rightarrow\{0,1\}$ будем говорить, что она сохраняет нуль, если

$$
f(0,0, \ldots, 0)=0 .
$$

Пусть для всякого $m \geq 1$ задана функция $\lambda_{0, m}$, сопоставляющая каждому вектору $\mathbf{t} \in\{0,1\}^{m}$ сохраняющую нуль функцию

$$
\lambda_{0, m}(\mathbf{t})=f_{\mathbf{t}}: R^{m} \rightarrow\{0,1\}
$$

со свойством $\left(\left\|f_{\mathbf{t}}\right\|, \tau\right)=1$. Чтобы задать функцию $\beta: \Omega \rightarrow\{0,1\}$, необходимо и достаточно задать ее на всяком $\Omega_{m}, m=1,2, \ldots$ (см. (6)).

Рассмотрим алгоритм вычисления значения $\beta(z)$ в точке $z \in \Omega_{m}$.

Алгоритм 1. (Вычисление значения $\beta(z)$ в точке $z \in \Omega_{m}$ ).

1. Вычислить разложение (35) состояния $z \in \Omega_{m}$ :

$$
z=z_{0}+z_{1}+\cdots+z_{m-1}, \quad z_{s} \in \operatorname{Ker} H_{s}(\rho), \quad s=0,1, \ldots, m-1 .
$$

2. Вычислить $\mathbf{t}=$ Tуре $z$ и найти функияию $f_{\mathbf{t}}=\lambda_{0, m}(\mathbf{t})$.

3. Вычислить

$$
\beta(z)=f_{\mathbf{t}}(v(0), v(1), \ldots, v(m-1)),
$$

где $v \in L_{R}(F)$ - ЛРП, ассочиированная с z в смысле равенства (20).

Фиксируя различными способами ряд

$$
\lambda_{0}=\left(\lambda_{0,1}, \lambda_{0,2}, \lambda_{0,3}, \ldots\right),
$$

будем получать разные функции $\beta$. Множество всех функций $\beta$, которые можно получить с помощью алгоритма 1 путем фиксации $\lambda_{0}$, обозначим через $B_{0}$.

При обосновании теоремы 3 было показано, что существует такая функция $\alpha: \mathbb{N} \rightarrow \mathbb{R}$, что

$$
\alpha(m)=o(1), \quad m \rightarrow \infty,
$$

и доля состояний $(0) \in \Omega_{m}$, для которых не выполняется совокупность условий

$$
\varepsilon_{0}=1 \quad \text { и } \quad\left(\varepsilon_{1}, 2 \varepsilon_{2}, 3 \varepsilon_{3}, \ldots,(m-1) \varepsilon_{m-1}, m\right)=1,
$$

не превосходит величины $\alpha(m)$. 
Ввиду (23) все состояния ж( 0$) \in \Omega_{m}$ со свойством

$$
T(u) \neq T(w),
$$

где $u \in L_{R}(F, F)$ и $u[\mathfrak{F}]=w(0)$, не удовлетворяют условиям (38). Таким образом, для любого $m \geq 1$ доля состояний ж( $(0) \in \Omega_{m}$ со свойством (39) не превосходит $\alpha(m)$. Принимая во внимание (37) и определение оптимальной функции самоуправления, получаем следующее утверждение.

Утверждение 3. Все функции самоуправления $\beta$ из класса $B_{0}$ оптимальныл.

Таким образом, построен класс $B_{0}$ оптимальных функций самоуправления. Его можно расширить, если изменить способ выбора функции $f$, фигурирующей в (21). Алгоритм 1 сопоставляет однотипным состояниям $z$ одинаковые функции $f$. Таким образом, однотипным циклам $C \subset L_{R}(F, F)$ сопоставляются одинаковые функции $f$. Следующий алгоритм разным циклам $C$ сопоставляет, вообще говоря, разные функции $f$.

Пусть для всякого $m \geq 1$ задана функция $\lambda_{1, m}$, сопоставляющая каждому циклу $C \subset L_{R}(F, F)$ некоторую сохраняющую нуль функцию

$$
\lambda_{1, m}(C)=f: R^{m} \rightarrow\{0,1\}
$$

со свойством $(\|f\|, \tau)=1$. Следующий алгоритм вычисляет значение $\beta(z)$ для всякого $z \in \Omega_{m}, m=1,2, \ldots$

Алгоритм 2. (Вычисление значения $\beta(z)$ в точке $z \in \Omega_{m}$.)

1. Вычислить компоненту $z_{0}$ из разложения (35) состояния $z \in \Omega_{m}$.

2. Пусть $C$ - иикл, которому принадлежит рекуррента $u \in L_{R}(F, F)$, определенная равенством $u[\mathfrak{F}]=z$. Найти функциюю $f=\lambda_{1, m}(C)$.

3. Вычислить

$$
\beta(z)=f(v(0), v(1), \ldots, v(m-1)),
$$

где $v \in L_{R}(F)$ - ЛРП, ассоииированная с $z$ в смысле равенства (20).

На данный момент не известно эффективного способа определить, какому из циклов принадлежит данная рекуррента $u \in L_{R}(F, F)$. Поэтому нет возможности эффективно осуществить шаг 2 алгоритма 2. Тем не менее, алгоритм 2 описывает более широкий (по сравнению с $B_{0}$ ) класс оптимальных функций самоуправления. 
Обозначим через $B_{1}$ класс функций самоуправления $\beta$, которые можно получить при помощи алгоритма 2 путем фиксации ряда

$$
\lambda_{1}=\left(\lambda_{1,1}, \lambda_{1,2}, \lambda_{1,3}, \ldots\right) \text {. }
$$

Ясно, что $B_{1} \supset B_{0}$. Аналогично утверждению 3 доказывается следующий факт.

Утверждение 4. Все функиии самоуправления $\beta$ из класса $B_{1}$ оптимальньл.

Рассмотрим класс $B_{2}$, состоящий из функций $\beta: \Omega \rightarrow\{0,1\}$, для каждой из которых существует такая функция $\beta^{\prime} \in B_{1}$, что $\beta(z)=\beta^{\prime}(z)$ почти для всех $z \in \Omega$. Очевидно, что $B_{2} \supset B_{1}$. Оказывается, в случае $R=\mathbb{Z}_{2}$ все оптимальные функции самоуправления принадлежат классу $B_{2}$. Докажем это.

Всюду далее в этом параграфе $q=2$, т. е. $R=\mathbb{Z}_{2}$. Пусть $\beta: \Omega \rightarrow$ $\rightarrow\{0,1\}-$ некоторая функция самоуправления, - последовательность (3) состояний автомата $\mathfrak{A}^{\beta}$, а управляющая последовательность $\delta$ определена равенством (1). Носителем $S p(w)$ последовательности ш будем называть множество состояний, принадлежащих этой последовательности.

Согласно (12), верно равенство $T(I)=\tau^{2}$. Очевидна следующая лемма.

Лемма 2. Если $T(w)=T(I)$, то для всякой пары

$$
\left(l_{0}, l_{1}\right) \in\{0,1, \ldots, \tau-1\} \times\{0,1, \ldots, \tau-1\}
$$

сущуествует единственное $i \in\left\{0,1, \ldots, \tau^{2}-1\right\}$ со свойством

$$
w(i)=\varphi_{0}^{l_{0}} \varphi_{1}^{l_{1}}(\omega(0)) .
$$

Согласно [1], реверсивной называется периодическая последовательность, у которой длина подхода равна 0.

Лемма 3 ( [6, теорема 1 и лемма 7]). Если $T(w)=T(I)$, то последовательность ш реверсивна и ограничение функции $\beta$ на $S p($ () инвариантно относительно автоморфизма $\rho$ :

$$
\forall z \in \operatorname{Sp}(w): \beta(z)=\beta(\rho(z))
$$

Обозначим через $\|\delta\|$ количество единиц на цикле управляющей последовательности $\delta$.

Лемма 4. Если $T(w)=T(I)$, то $\delta$ реверсивна, $T(\delta)=\tau u(\|\delta\|, \tau)=1$. 
Доказательство. Реверсивность последовательности $\delta$ следует из (1) и реверсивности последовательности $w$. Пусть $i \geq 0$. Если $l_{0}-$ число нулей, а $l_{1}$ - число единиц на отрезке $\delta(0,1, \ldots, i-1)$, то справедливо равенство (40). Ввиду (1) и равенства $l_{0}+l_{1}=i$ получаем

$$
\delta(i)=\beta(w(i))=\beta\left(\varphi_{0}^{l_{0}} \varphi_{1}^{l_{1}}(w(0))\right)=\beta\left(\rho^{l_{1}} \varphi_{0}^{i}(w(0))\right) .
$$

Согласно лемме 2 имеем: $\varphi_{0}^{i}(w(0)) \in S p(w)$. В силу леммы 3 верно равенство

$$
\delta(i)=\beta\left(\varphi_{0}^{i}(w(0))\right),
$$

гарантирующее, что $T(\delta) \mid \tau$.

Для доказательства равенства $T(\delta)=\tau$ достаточно показать, что $(\|\delta\|, \tau)=1$. От противного: пусть $(\|\delta\|, \tau)=d>1$ и отрезок $\delta(0,1, \ldots, \tau-1)$ содержит $d k_{0}$ нулей и $d k_{1}$ единиц. Поскольку $T(\delta) \mid \tau$, для всякого $j \geq 0$ имеет место равенство

$$
w(j \tau)=\varphi_{0}^{j d k_{0}} \varphi_{1}^{j d k_{1}}(w(0))
$$

Отсюда

$$
T(w) \mid \frac{\tau}{d} \cdot \tau<\tau^{2}=T(I),
$$

что противоречит условию. $\triangleright$

Из леммы 4 и теоремы 2 вытекает следующее утверждение.

Утверждение 5. Пусть состояние (см. (36)). Тогда для равенства $T(w)=T(I)$ необходимо и достаточно выполнение условий

$$
T(\delta)=\tau, \quad(\|\delta\|, \tau)=1 \quad u \quad\left(\varepsilon_{1}, 2 \varepsilon_{2}, 3 \varepsilon_{3}, \ldots,(m-1) \varepsilon_{m-1}, m\right)=1 .
$$

Обозначим через $V_{m}$ множество всех векторов $\left(\varepsilon_{0}, \varepsilon_{1}, \ldots, \varepsilon_{m-1}\right) \in$ $\in\{0,1\}^{m}$, обладающих свойствами (38). Для всякого цикла $C \subset L_{R}(F, F)$ положим

$$
C[\mathfrak{F}]=\{u[\mathfrak{F}] \mid u \in C\} .
$$

Следующий результат - центральный в этом параграфе.

Теорема 5. Пусть $R=\mathbb{Z}_{2}, \beta: \Omega \rightarrow\{0,1\}, m \geq 1$. Следуюшие утверждения эквивалентны:

1) для всякого состояния w $(0) \in \Omega_{m}$ со свойством Туре $(0) \in V_{m}$ верно равенство $T(w)=T(I)$; 
2) для любого ичикла $C \subset L_{R}(F, F)$ со свойством Туре $C \in V_{m}$ сущеествует такая булева функция $f\left(x_{1}, x_{2}, \ldots, x_{m}\right)$, что $f(0,0, \ldots, 0)=0$, $(\|f\|, \tau)=1 u$

$$
\forall z \in C[\mathfrak{F}]: \quad \beta(z)=f(v(0), v(1), \ldots, v(m-1)),
$$

где $v \in L_{R}(F)$ - ЛРП, ассочиированная с z в смысле равенства (20).

Доказательство. 1) $\Rightarrow$ 2). Пусть

$$
\text { Type } C \in V_{m}, \quad u \in C, \quad w(0)=u[\mathfrak{F}] .
$$

Тогда $T(w)=T(I)$. Согласно утверждению 5 верны равенства

$$
T(\delta)=\tau \quad \text { и } \quad(\|\delta\|, \tau)=1 .
$$

Это означает, что для всякого $i \geq 0$ знак $\delta(i)$ полностью определяется отрезком

$$
v(i, i+1, \ldots, i+m-1)
$$

последовательности $v$, ассоциированной с $(0)$ :

$$
\forall i \geq 0: \quad \delta(i)=f(v(i), v(i+1), \ldots, v(i+m-1)),
$$

где $f\left(x_{1}, x_{2}, \ldots, x_{m}\right)$ - некоторая булева функция. Так как $v-$ ЛРП максимального периода (ЛРП МП), каждая ненулевая $m$-грамма присутствует на ее цикле один раз. Поэтому можно считать, что $f(0,0, \ldots, 0)=0$, и из условия $(\|\delta\|, \tau)=1$ вытекает равенство $(\|f\|, \tau)=1$. Осталось заметить, что $C[\mathfrak{F}]=S p(w)$, и ЛРП $x^{i} v$ ассоциирована с $w(i), i=0,1,2, \ldots$

$2) \Rightarrow 1)$. Пусть начальное состояние $w(0) \in \Omega_{m}$ таково, что Туре $\in V_{m}$. По условию существует такая булева функция $f\left(x_{1}, x_{2}, \ldots, x_{m}\right)$, что $f(0,0, \ldots, 0)=0,(\|f\|, \tau)=1$, и выполняется равенство (42), где $v-$ ЛРП, ассоциированная с $(0)$. Так как $v-$ ЛРП МП, каждая ненулевая $m$-грамма присутствует на ее цикле один раз. Поэтому выполняются условия (41) и, согласно утверждению 5 , верно равенство $T(\varpi)=T(I)$.

Обозначим через $B$ множество всех функций самоуправления $\beta: \Omega \rightarrow$ $\rightarrow\{0,1\}$, удовлетворяющих условию

$$
\forall m \geq 1, w(0) \in \Omega_{m}:\left(\text { Type } w(0) \in V_{m}\right) \Rightarrow(T(w)=T(I)) .
$$

При доказательстве теоремы 3 было показано, что число состояний ж(0) $\in$ $\in \Omega_{m}$ со свойством Туре деления класса $B_{1}$ (см. алгоритм 2) следует, что для всякой функции $\beta \in B$ 
существует такая функция $\beta^{\prime} \in B_{1}$, что $\beta(z)=\beta^{\prime}(z)$ почти для всех $z \in \Omega$. Иными словами, $B \subseteq B_{2}$.

Пусть $\beta: \Omega \rightarrow\{0,1\}-$ оптимальная функция самоуправления. Доля состояний $(0) \in \Omega_{m}$ со свойствами

$$
\text { Туре }(0) \in V_{m} \quad \text { и } \quad T(w) \neq T(u),
$$

где 2-ЛРП $u \in L_{R}(F, F)$ определена равенством $u[\mathfrak{F}]=w(0)$, есть $o(1)$ при $m \rightarrow \infty$. Ввиду (23) из включения Туре ж(0) $\in V_{m}$ следует, что $T(u)=T(I)$. Поэтому доля состояний ж( $(0) \in \Omega_{m}$ со свойствами

$$
\text { Туре } w(0) \in V_{m} \quad \text { и } \quad T(w) \neq T(I)
$$

есть $o(1)$ при $m \rightarrow \infty$. Следовательно, существует функция $\beta^{\prime} \in B$, совпадающая с $\beta$ почти во всех точках $z \in \Omega$. Учитывая, что $B \subseteq B_{2}$, получаем искомый результат.

Следствие 4. Если $R=\mathbb{Z}_{2}$ и функиия самоуправления $\beta$ оптимальна, mo $\beta \in B_{2}$.

\section{4. Выбор функции выхода}

Пусть $R=G F(q), m \geq 1, F(x)=F_{m}(x)-m$-й многочлен из ряда (7), $\beta \in B_{1}$ (см. параграф 3.), $\Psi_{m}-$ множество ненулевых линейных функций $\psi: \Omega_{m} \rightarrow R:$

$$
\forall z \in \Omega_{m}: \psi(z)=\sum_{k=1}^{t} d_{k} z_{i_{k}, j_{k}},
$$

где $t \in\left\{1,2, \ldots, m^{2}\right\}, d_{k} \in R^{*}$ и $\left(i_{k}, j_{k}\right) \in \mathfrak{F}$ для $k=1,2, \ldots, t, z_{i, j} \in R-$ элемент, стоящий на пересечении $i$-й строки и $j$-го столбца матрицы $z$ (нумерация строк и столбцов ведется с нуля). Цель этого параграфа - описать линейные функции выхода $\psi$ вида (44), для которых имеет место импликация

$$
(T(w)=T(I)) \Rightarrow(T(\gamma)=T(w)),
$$

где $I=\left(F\left(x_{0}\right), F\left(x_{1}\right)\right)_{R_{2}}$, а $ш$ и $\gamma-$ соответственно последовательность состояний и выходная последовательность самоуправляемого 2-ЛРС $\mathfrak{A}^{\beta}$. Напомним некоторые обозначения и результаты из работы [4]. 
Напомним (см. параграф 2), что поле $Q=G F\left(q^{m}\right)$ можно рассматривать как расширение поля $R$ корнем $\theta$ многочлена $F(x): Q=R[\theta]$. Обозначим через $\operatorname{tr}$ функцию "след" из $Q$ в $R$ (см., например, [1]):

$$
\forall x \in Q: \quad \operatorname{tr}(x)=\sum_{i=0}^{m-1} x^{q^{i}} .
$$

Для всякого $s \in\{0,1, \ldots, m-1\}$ рассмотрим функцию $\pi_{s}: Q \rightarrow \Omega_{m}$, заданную равенством

$$
\forall x \in Q: \quad \pi_{s}(x)=\sum_{i=0}^{m-1} \sum_{j=0}^{m-1} \operatorname{tr}\left(\theta^{q^{s} i+j} x\right) e_{i, j} .
$$

Согласно [4, утверждение 3], верны равенства

$$
\operatorname{Ker} H_{s}(\rho)=\pi_{s}(Q), \quad s=0,1, \ldots, m-1 .
$$

Поэтому имеет место следующее разложение пространства $\Omega_{m}$ в прямую сумму инвариантных подпространств:

$$
\Omega_{m}=\pi_{0}(Q) \dot{+} \pi_{1}(Q) \dot{+} \ldots \dot{+} \pi_{m-1}(Q),
$$

и в разложении (35) $z_{s} \in \pi_{s}(Q), s=0,1, \ldots, m-1$.

Рассмотрим линейную функцию выхода $\psi \in \Psi_{m}$. Если

$$
w(i)=w_{0}(i)+w_{1}(i)+\cdots+w_{m-1}(i)
$$

- разложение вида (35), то

$$
w_{s}(i) \in \pi_{s}(Q), \quad s=0,1, \ldots, m-1,
$$

и, согласно (2), верно равенство

$$
\gamma=\gamma_{0}+\gamma_{1}+\cdots+\gamma_{m-1}
$$

где $\gamma_{s}(i)=\psi\left(w_{s}(i)\right), i \geq 0, s=0,1, \ldots, m-1$.

Пусть $s \in\{0,1, \ldots, m-1\}$. Исследуем последовательность $\gamma_{s}$. С этой целью рассмотрим многочлен $\chi_{s}(x) \in R[x]$, определенный равенством

$$
\chi_{s}(x)=\sum_{k=1}^{t} d_{k} x^{q^{s} i_{k}+j_{k}} .
$$


Лемма 5. Пусть $T(w)=T(I)$ и $\varepsilon_{s}=1$. Для равенства $\gamma_{s}=0$ необходимо и достаточно выполнение соотномения $F(x) \mid \chi_{s}(x)$.

Доказательство. По условию $\varepsilon_{s}=1$. Ввиду (47) существует такой элемент $a \in Q \backslash\{0\}$, что $w_{s}(0)=\pi_{s}(a)$. Следовательно, в силу (46) верно равенство

$$
w_{S}(0)=\sum_{i=0}^{m-1} \sum_{j=0}^{m-1} v\left(q^{s} i+j\right) e_{i, j},
$$

где $v \in L_{R}(F)$ - ЛРП МП. Рассмотрим ЛРП $v^{\prime}=\chi_{s} \cdot v \in L_{R}(F)$. Очевидно, что

$$
F(x) \mid \chi_{s}(x) \quad \Leftrightarrow \quad v^{\prime}=0 .
$$

Осталось доказать, что

$$
v^{\prime}=0 \quad \Leftrightarrow \quad \gamma_{s}=0 .
$$

Заметим, что $\gamma_{s}$ есть подпоследовательность последовательности $v^{\prime}$. Действительно, пусть $l \geq 0, i_{0}$ и $j_{0}-$ соответственно числа нулей и единиц на отрезке

$$
\delta(0,1, \ldots, l-1)
$$

Тогда

$$
w_{S}(l)=\sum_{i=0}^{m-1} \sum_{j=0}^{m-1}\left(x^{q^{s} i_{0}+j_{0}} v\right)\left(q^{s} i+j\right) e_{i, j}
$$

и верна цепочка равенств

$$
\gamma_{s}(l)=\psi\left(w_{s}(l)\right)=\left(\sum_{k=1}^{t} d_{k} x^{q^{s} i_{k}+j_{k}} v\right)\left(q^{s} i_{0}+j_{0}\right)=v^{\prime}\left(q^{s} i_{0}+j_{0}\right) .
$$

В частности, если $v^{\prime}=0$, то $\gamma_{s}=0$.

Обратно: пусть $\gamma_{s}=0$. Докажем, что $v^{\prime}=0$. Если $s=0$, то в силу (49)

$$
\gamma_{s}(l)=v^{\prime}\left(i_{0}+j_{0}\right)=v^{\prime}(l)
$$

для всякого $l \geq 0$. Тогда $v^{\prime}=\gamma_{s}=0$.

Пусть $s \geq 1$. Рекуррента $v^{\prime} \in L_{R}(F)$ представляется функцией «след» (см., например, [1]):

$$
\exists \alpha \in Q \backslash\{0\}: \forall i \geq 0: v^{\prime}(i)=\operatorname{tr}\left(\alpha \theta^{i}\right) .
$$


Пусть 2-ЛРП $u \in L_{R}(F, F)$ определена равенством $u[\mathfrak{F}]=w(0), C \subseteq$ $\subseteq L_{R}(F, F)$ - цикл, содержащий рекурренту $u, f=\lambda_{1, m}(C)$ (см. параграф 3). Если $k_{f}=\|f\|$, то ввиду (49) и (50) имеем

$$
\forall i \geq 0: \quad 0=\gamma_{s}(i \tau)=v^{\prime}\left[\left(q^{s}\left(\tau-k_{f}\right)+k_{f}\right) i\right]=\operatorname{tr}\left(\alpha \theta^{-k_{f}\left(q^{s}-1\right) i}\right)=\operatorname{tr}\left(\alpha \vartheta_{s}^{i}\right),
$$

где $\vartheta_{s}=\theta^{-k_{f}\left(q^{s}-1\right)} \in Q-$ элемент с минимальным многочленом $G_{s}(x)$ (теорема 1). Так как $\operatorname{deg} G_{s}(x)=m$, система

$$
\vartheta_{s}^{0}, \vartheta_{s}^{1}, \ldots, \vartheta_{s}^{m-1}
$$

линейно независима над $R$. Следовательно, система линейных уравнений

$$
\operatorname{tr}\left(\alpha \vartheta_{s}^{i}\right)=0, \quad i=0,1, \ldots, m-1,
$$

имеет единственное решение $\alpha=0$. Тогда в силу (50) верно равенство $v^{\prime}=0$. $\triangleright$

Вычислим характеристический многочлен и период ЛРП $\gamma_{s}$ в ситуации

$$
\text { Tуре } ш(0) \in V_{m}, \quad s \geq 1 \quad \text { и } \quad \gamma_{s} \neq 0 .
$$

Так как $\beta \in B_{1}$, число $k_{f}=\|f\|$ взаимно просто с $\tau$. В силу (16) верно равенство $\sigma=\rho^{k_{f}}$. В базисе $\boldsymbol{e}^{\downarrow}$ (см. параграф 2) автоморфизм $\sigma$ имеет матрицу

$$
A=S(F)^{-k_{f}} \otimes S(F)^{k_{f}} .
$$

Нетрудно показать (см., например, [4]), что вторая нормальная форма [1] матрицы $A$ имеет вид

$$
\operatorname{Diag}\left(E, S\left(G_{1}\right), S\left(G_{2}\right), \ldots, S\left(G_{m-1}\right)\right),
$$

где $E-$ единичная матрицами размерами $m \times m$.

Выберем и зафиксируем обратимую матрицу $C \in R_{m^{2}, m^{2}}$ со свойством

$$
C A C^{-1}=\operatorname{Diag}\left(E, S\left(G_{1}\right), S\left(G_{2}\right), \ldots, S\left(G_{m-1}\right)\right) .
$$

Автоморфизм $\sigma$ осуществляет регулярную $\tau$-выборку из последовательности $w_{s}$ :

$$
\forall d \in\{0,1, \ldots, \tau-1\}: \forall i \geq 0: \sigma^{i}\left(w_{s}(d)\right)=w_{s}(d+i \tau) .
$$

Пусть $\vec{x}=\left(x_{1}, x_{2}, \ldots, x_{m}\right) \in R^{m}$. Обозначим через $(\overrightarrow{0}, \vec{x}, \overrightarrow{0})$ вектор длины $m^{2}$ вида

$$
\left(0,0, \ldots, 0, x_{1}, x_{2}, \ldots, x_{m}, 0,0, \ldots, 0\right)
$$


где элементу $x_{1}$ предшествуют $m s$ нулей. В силу (52) существует такая ЛРП $v_{s, d} \in L_{R}\left(G_{s}\right)$, что

$$
\forall i \geq 0: \vec{\omega}_{s}(d+i \tau)=\left(\overrightarrow{0}, v_{s, d}(i, i+1, \ldots, i+m-1), \overrightarrow{0}\right) \cdot C,
$$

где $\vec{\mho}_{s}(d+i \tau)$ - строка координат вектора $w_{s}(d+i \tau)$ в базисе $\boldsymbol{e}^{\downarrow}$.

Пусть $\gamma_{s}^{(d)}-$ регулярная $(d, \tau)$-выборка из последовательности $\gamma_{s}$. Тогда

$$
\forall i \geq 0: \gamma_{s}^{(d)}(i)=\psi\left[w_{s}(d+i \tau)\right] .
$$

Ввиду (53) существует многочлен $g_{s}(x) \in R[x]$ со свойствами $\operatorname{deg} g_{s}(x) \leq$ $\leq m-1$ и

$$
\gamma_{s}^{(d)}=g_{s}(x) \cdot v_{s, d} .
$$

Соотношение (55) справедливо для любого $d \in\{0,1, \ldots, \tau-1\}$. Так как $\gamma_{s} \neq 0$, имеем $\varepsilon_{s}=1$ и $g_{s}(x) \neq 0$. Равенство $\varepsilon_{s}=1$ означает, что

$$
\forall d \in\{0,1, \ldots, \tau-1\}: w_{s}(d) \neq 0 .
$$

Отсюда и из (53) следует, что

$$
\forall d \in\{0,1, \ldots, \tau-1\}: d v_{s, d} \neq 0 .
$$

Из неравенства $g_{s}(x) \neq 0$ вытекает, что $\left(g_{s}(x), G_{s}(x)\right)=1$. В силу (55) многочлен $G_{s}(x)$ является минимальным для регулярной $\tau$-выборки $\gamma_{s}^{(d)}$, $d=0,1, \ldots, \tau-1$.

Напомним (см. теорему 2), что $\tau_{s}=\tau /\left(q^{(m, s)}-1\right)$.

Лемма 6. Если выполнены условия (51), то

$$
G_{s}\left(x^{\tau}\right) \gamma_{s}=0 \quad u \quad T\left(\gamma_{s}\right)=\tau \tau_{s} .
$$

Доказательство. Первое равенство уже доказано. Докажем второе.

По последовательности $\gamma_{s}$ однозначно восстанавливается всякая регулярная $\tau$-выборка $\gamma_{s}^{(d)}, d=0,1, \ldots, \tau-1$. По последовательности $\gamma_{s}^{(d)}$ при помощи (55) однозначно восстанавливается отрезок $v_{s, d}(0,1, \ldots, m-1)$, а значит, и вся рекуррента $v_{s, d} \in L_{R}\left(G_{s}\right)$. По рекуррентам $v_{s, d}, d=0,1, \ldots, \tau-1$, при помощи (53) однозначно восстанавливается всякая регулярная $\tau$-выборка из $w_{s}$, а следовательно, и вся последовательность $w_{s}$. Поэтому $T\left(\gamma_{s}\right)=T\left(w_{s}\right)$.

Ввиду (53) период всякой регулярной $(d, \tau)$-выборки из шs равен $T\left(v_{s, d}\right)$. В силу (56) имеем $T\left(v_{s, d}\right)=T\left(G_{s}\right)=\tau_{s}$. Поэтому период всякой 
регулярной $\tau$-выборки из $\varpi_{s}$ равен $\tau_{s}$. Согласно [4, утверждение 7], верно соотношение $\tau \mid T\left(w_{s}\right)$. Поэтому

$$
\tau_{s}=T\left(w_{s}\right) / \tau \quad \text { и } \quad T\left(\gamma_{s}\right)=T\left(w_{s}\right)=\tau \tau_{s} . \triangleright
$$

Типом функции выхода $\psi: \Omega_{m} \rightarrow R$ назовем вектор

$$
\text { Type } \psi=\left(\varepsilon_{0}^{\prime}, \varepsilon_{1}^{\prime}, \ldots, \varepsilon_{m-1}^{\prime}\right),
$$

где

$$
\forall s \in\{0,1, \ldots, m-1\}: \varepsilon_{s}^{\prime}= \begin{cases}0, & \text { если } F(x) \mid \chi_{s}(x), \\ 1 & - \text { в противном случае. }\end{cases}
$$

Следующий результат - центральный в этом параграфе.

Tеорема 6. Пусть $R=G F(q), \beta \in B_{1}$, Туре $w(0)=\left(\varepsilon_{0}, \varepsilon_{1}, \ldots, \varepsilon_{m-1}\right) \in$ $\in V_{m}, \psi \in \Psi_{m}$, Type $\psi=\left(\varepsilon_{0}^{\prime}, \varepsilon_{1}^{\prime}, \ldots, \varepsilon_{m-1}^{\prime}\right), a_{s}=\varepsilon_{s} \varepsilon_{s}^{\prime}, s=0,1, \ldots, m-1$. Тогда

$$
T(\gamma)=\left[\tau^{a_{0}},\left(\tau \tau_{1}\right)^{a_{1}},\left(\tau \tau_{2}\right)^{a_{2}}, \ldots,\left(\tau \tau_{m-1}\right)^{a_{m-1}}\right],
$$

где $\tau_{s}=\tau /\left(q^{(m, s)}-1\right), s=1,2, \ldots, m-1$. Равенство $T(\gamma)=T(I)$ выполняется тогда и только тогда, когда $\left(a_{1}, 2 a_{2}, \ldots,(m-1) a_{m-1}, m\right)=1$.

Доказательство. Так как $\beta \in B_{1}$ и Туре $(0) \in V_{m}$, верно равенство $T(w)=T(I)$. По лемме 5 и определению (57) значение $a_{s}=\varepsilon_{s} \varepsilon_{s}^{\prime}-$ индикатор условия $\gamma_{s} \neq 0, s=0,1, \ldots, m-1$. Если $\gamma_{0} \neq 0$, то ввиду (20) минимальный многочлен этой последовательности равен $F(x)$, и, следовательно, $T\left(\gamma_{0}\right)=\tau$.

В случае $m=1$ формула (58) очевидна. Пусть $m \geq 2$. Многочлены

$$
G_{1}(x), G_{2}(x), \ldots, G_{m-1}(x)
$$

попарно взаимно просты, неприводимы и имеют степень $m$. Поэтому многочлены

$$
F(x), G_{1}\left(x^{\tau}\right), G_{2}\left(x^{\tau}\right), \ldots, G_{m-1}\left(x^{\tau}\right)
$$

также попарно взаимно просты. Теперь формула (58) следует из разложения (48) и леммы 6.

Докажем второе утверждение теоремы. Пусть

$$
\left\{j_{1}, j_{2}, \ldots, j_{k}\right\}=\left\{j \in\{1,2, \ldots, m-1\}: a_{j}=1\right\} .
$$

В силу (58) имеем

$$
T(\gamma)=\frac{\tau^{2}}{q^{d}-1}
$$


где $d=\left(j_{1}, j_{2}, \ldots, j_{k}, m\right)$. Поэтому $T(\gamma)=T(I)$ тогда и только тогда, когда $d=1$, т. е. $\left(a_{1}, 2 a_{2}, \ldots,(m-1) a_{m-1}, m\right)=1$.

В связи с теоремой 6 возникает вопрос: как велико множество линейных функций $\psi \in \Psi_{m}$, гарантирующих выполнение импликации (45)? На этот вопрос отвечает следующее утверждение.

Следствие 5. Если $\beta \in B_{1} u$ Туре $(0) \in V_{m}$, то доля линейных функиий выхода $\psi$, для которых $T(\gamma)<T(I)$, есть о(1) при $m \rightarrow \infty$.

Доказательство. Пусть

$$
L_{m}=\Psi_{m} \cup\{0\}
$$

- множество всех линейных функций $\psi: \Omega_{m} \rightarrow R$. Оценим сначала число функций $\psi \in L_{m}$, для которых $T\left(\gamma_{0}\right)<T\left(w_{0}\right)$. Поскольку $F(x) \cdot \gamma_{0}=0$, то либо $\gamma_{0}=0$, либо $T\left(\gamma_{0}\right)=\tau=T\left(w_{0}\right)$. По лемме 5 необходимо оценить число функций $\psi \in L_{m}$, для которых $F(x) \mid \chi_{0}(x)$.

Согласно (20) всякая матрица $z \in \pi_{0}(Q)$ имеет следующий вид:

$$
z=\left(\begin{array}{ccccc}
a_{0} & a_{1} & \ldots & a_{m-2} & a_{m-1} \\
a_{1} & a_{2} & \ldots & a_{m-1} & a_{m} \\
\vdots & \vdots & \ddots & \vdots & \vdots \\
a_{m-2} & a_{m-1} & \ldots & a_{2 m-4} & a_{2 m-1} \\
a_{m-1} & a_{m} & \ldots & a_{2 m-1} & a_{2 m-2}
\end{array}\right),
$$

т. е. на пересечении $i$-й строки и $j$-го столбца стоит элемент $a_{i+j}$ (нумерация строк и столбцов идет с нуля). Отсюда вытекают следующие факты.

1. Имеет место равенство

$$
\left\{\chi_{0}(x) \mid \psi \in L_{m}\right\}=\{\chi(x) \in R[x] \mid \operatorname{deg} \chi(x) \leq 2 m-2\}
$$

(здесь предполагается, что функции $\psi=0$ соответствует нулевой многочлен $\left.\chi_{0}(x)\right)$. В частности, всего существует $q^{2 m-1}$ многочленов $\chi_{0}(x)$. Из них $q^{m}$ многочленов делятся на $F(x)$.

2. Пусть функция $\psi \in L_{m}$ имеет вид

$$
\psi(z)=\sum_{i=0}^{m-1} \sum_{j=0}^{m-1} d_{i, j} z_{i, j} .
$$


Тогда коэффициент при $x^{k}$ в многочлене $\chi_{0}(x)$ равен сумме коэффициентов функции $\psi$, соответствующих элементам $k$-й побочной диагонали матрицы (59):

$$
\operatorname{Coef}_{x^{k}}\left(\chi_{0}(x)\right)=\sum_{(i, j): i+j=k} d_{i, j} .
$$

Это означает, что одному многочлену $\chi_{0}(x)$ соответствует

$$
q^{(m-1)+2 \sum_{i=1}^{m-2} i}=q^{(m-1)^{2}}
$$

функций $\psi \in L_{m}$.

Из пп. 1 и 2 следует, что число функций $\psi \in L_{m}$ со свойством $F(x) \mid \chi_{0}(x)$ (или, что то же самое, со свойством $T\left(\gamma_{0}\right)<T\left(w_{0}\right)$ ) равно

$$
q^{m+(m-1)^{2}}=q^{m^{2}-m+1} .
$$

Пусть теперь $s \in\{1,2, \ldots, m-1\}, C$ - матрица из равенства (52), и $C_{s}$ - подматрица матрицы $C$, стоящая в строках $m s+1, m s+2, \ldots, m(s+1)$ и столбцах $1,2, \ldots, m^{2}$. Занумеруем столбцы матрицы $C_{s}$ парами $(i, j) \in \mathfrak{F}$ :

$$
C_{s}=\left(c_{0,0}^{\downarrow}, c_{0,1}^{\downarrow}, \ldots, c_{0, m-1}^{\downarrow}, c_{1,0}^{\downarrow}, c_{1,1}^{\downarrow}, \ldots, c_{1, m-1}^{\downarrow}, \ldots\right) .
$$

В силу (44), (53) и (54) для всякого $d \in\{0,1, \ldots, \tau-1\}$ имеем

$$
\forall i \geq 0: \gamma_{s}^{(d)}(i)=l_{s}\left(v_{s, d}(i, i+1, \ldots, i+m-1)\right),
$$

где $l_{s}(\vec{x})=\vec{x} \cdot \sum_{k=1}^{t} d_{k} c_{i_{k}, j_{k}}^{\downarrow}$.

Если $\varepsilon_{s}=0$, то, очевидно, $T\left(\gamma_{s}\right)=T\left(w_{s}\right)=1$. Если $\varepsilon_{s}=1$, то

$$
T\left(\gamma_{s}\right)=T\left(w_{s}\right) \Leftrightarrow l_{s} \neq 0 \Leftrightarrow \sum_{k=1}^{t} d_{k} c_{i_{k}, j_{k}}^{\downarrow} \neq 0^{\downarrow} .
$$

Поясним цепочку (62). Вторая эквивалентность очевидна. Рассмотрим первую эквивалентность:

$$
T\left(\gamma_{s}\right)=T\left(w_{s}\right) \Leftrightarrow l_{s} \neq 0 .
$$

Импликация «слева направо» следует из (61). Докажем импликацию «справа налево». По условию Туре $(0) \in V_{m}$ и $s \geq 1$. Если $v_{s, 0}=0$, то, согласно (53), $w_{s}(0)=0$, что противоречит равенству $\varepsilon_{s}=1$. Следовательно, $v_{s, 0} \neq 0$, и в силу $(61) \gamma_{s}^{(0)} \neq 0$. Тогда $\gamma_{s} \neq 0$, и выполняются условия (51). При 
доказательстве леммы 6 было показано, что условия (51) влекут равенство $T\left(\gamma_{s}\right)=T\left(w_{s}\right)$.

Очевидно, что rang $C_{s}=m$. Из (62) следует, что число функций $\psi \in L_{m}$, для которых $T\left(\gamma_{s}\right)<T\left(w_{s}\right)$, равно числу решений системы $C_{s} x^{\downarrow}=0 \downarrow$, т. е. $q^{m^{2}-m}$. С учетом (60) получаем, что доля $\Delta$ функций выхода $\psi \in L_{m}$, для которых $T(\gamma)<T(w)$, удовлетворяет цепочке неравенств

$\Delta \leq \frac{1}{\left|L_{m}\right|}\left\{q^{m^{2}-m+1}+\sum_{1 \leq s \leq m-1: \varepsilon_{s}=1} q^{m^{2}-m}\right\} \leq(m-1+q) q^{-m}=o(1), \quad m \rightarrow \infty$,

что и требовалось доказать. $\triangleright$

Следует заметить, что доказательство следствия 5 дает не только асимптотический результат о доле $\Delta$ функций выхода $\psi \in L_{m}$, для которых $T(\gamma)<T(w)$, но и верхнюю оценку этой доли: $\Delta \leq(m-1+q) q^{-m}$.

\section{5. Заключение}

Вернемся к цепочке неравенств (4):

$$
T(\gamma) \leq T(w) \leq T(u) \leq T(I) .
$$

Выводы по итогам работы:

1. Если $R$ - кольцо Галуа и $F$ - особый ММП (см. параграф 2), то почти все рекурренты $u \in L_{R}(F, F)$ принадлежат циклам максимально возможной длины: $T(u)=T(I)$.

2. Пусть $R=\mathbb{Z}_{2}$ и $T(u)=T(I)$. Условие $T(w)=T(u)$ накладывает жесткие ограничения на вид функции $\beta$ (см. теорему 5). Поэтому $T(w)<T(u)$ почти для всех функций самоуправления $\beta$.

3. Пусть $R=G F(q)$ и $T(w)=T(u)=T(I)$. Тогда почти все линейные функции выхода $\psi$ обеспечивают равенство $T(\gamma)=T($ w).

Полученные результаты позволяют осознанно выбирать параметры 2-ЛРС при его использовании в качестве генератора ПСП.

\section{6. Благодарность}

Автор выражает глубокую признательность профессору А. А. Нечаеву за постановку задачи и постоянное внимание к этой работе и А. М. Зубкову за ценные замечания по тексту статьи. 


\section{Список литературы}

1. Глухов М. М., Елизаров В. П., Нечаев А. А. Алгебра. - М.: Гелиос, 2003. $749 \mathrm{c}$.

2. Елизаров В. П. Конечные кольца. - М.: Гелиос, 2006. - 304 с.

3. Камловский О.В. Количество различных мультиграмм в линейных рекуррентных последовательностях над кольцами Галуа // Математические вопросы криптографии. - 2013. - Т. 4. Вып. 3. C. 49-82.

4. Козлитин O.A. 2-линейный регистр сдвига над кольцом Галуа четной характеристики // Математические вопросы криптографии. - 2012. Т. 3. Вып. 2. - С. 27-61.

5. Козлитин О.А. Параллельная декомпозиция неавтономных 2-линейных регистров сдвига // Математические вопросы криптографии. - 2011. T. 2. Вып. 3. - С. 5-29.

6. Козлитин О.А. Периодические свойства простейшего 2-линейного регистра сдвига // Дискретн. матем. - 2007. - Т. 19. Вып. 3. C. 51-78.

7. Козлитин О.А. Свойства выходной последовательности простейшего самоуправляемого 2-линейного регистра сдвига // Дискретная математика. - 2007. - Т. 19. Вып. 4. - С. 70-96.

8. Михайлов Д.А. Унитарные полилинейные регистры сдвига и их периоды // Дискретная математика. - 2002. - Т. 14. Вып. 1. C. 30-59.

9. Михалев А. В., Нечаев А. А. Цикловые типы семейств полилинейных рекуррент и датчики псевдослучайных чисел // Математические вопросы криптографии. - 2014. - Т. 5. Вып. 1. - С. 95-125.

10. Нечаев А. А. Многомерные регистры сдвига и сложность мультипоследовательностей // В сб.: Труды по дискретной математике, т. 6. - М.: Физматлит, 2003. - С. 150-165.

11. Нечаев А. А. Цикловые типы линейных подстановок над конечными коммутативными кольцами // Математический сборник. - 1993. - Т. 184. Вып. 3. - С. 21-56.

12. Сачков В.Н. Введение в комбинаторные методы дискретной математики. - М.: Наука, 1982. - 384 с.

13. Словарь криптографических терминов / под ред. Погорелова Б.А. и Сачкова В.Н. - М.: МЦНМО, 2006. - 94 с.

14. Фомичев В.М. Дискретная математика и криптология. - М.: ДиалогМИФИ, 2003. - 397 с. 
15. Kurakin V.L., Kuzmin A.S., Mikhalev A. V., Nechaev A.A. Linear recurring sequences over rings and modules // J. of Math. Sciences. - 1995. - V. 76. № 6. - P. 2793-2915.

16. McDonald B.R. Finite rings with identity. - New York: Dekker, 1974. $429 \mathrm{p}$.

17. Nomura T., Fukuda A. Linear recurring planes and two-dimensional cyclic codes // Electronic Commun. Japan. - 1971. - V. 54. №3. - P. 23-30.

18. Sakata $S$. Extension of the Berlekamp-Massey algorithm to N dimensions // Inform. and Comput. - 1990. - V.84. №2. - P. 207-239.

19. http://en.wikipedia.org/wiki/Salsa20. 